\title{
Improvements in Lower-Extremity Function Following a Rehabilitation Program With Patterned Electrical Neuromuscular Stimulation in Females With Patellofemoral Pain: A Randomized Controlled Trial
}

\author{
Neal R. Glaviano, Ashley N. Marshall, L. Colby Mangum, Joseph M. Hart, \\ Jay Hertel, Shawn Russell, and Susan Saliba
}

\begin{abstract}
Context: Patellofemoral pain (PFP) is a challenging condition, with altered kinematics and muscle activity as 2 common impairments. Single applications of patterned electrical neuromuscular stimulation (PENS) have improved both kinematics and muscle activity in females with PFP; however, the use of PENS in conjunction with a rehabilitation program has not been evaluated. Objective: To determine the effects of a 4-week rehabilitation program with PENS on lower-extremity biomechanics and electromyography (EMG) during a single-leg squat (SLS) and a step-down task (SDT) in individuals with PFP. Study Design: Double-blinded randomized controlled trial. Setting: Laboratory. Patients of Other Participants: Sixteen females with PFP (age 23.3 [4.9] y, mass 66.3 [13.5] kg, height 166.1 [5.9] cm). Intervention: Patients completed a 4-week supervised rehabilitation program with or without PENS. Main Outcome Measures: Curve analyses for lower-extremity kinematics and EMG activity (gluteus maximus, gluteus medius, vastus medialis oblique, vastus lateralis, biceps femoris, and adductor longus) were constructed by plotting group means and $90 \%$ confidence intervals throughout $100 \%$ of each task, before and after the rehabilitation program. Mean differences (MDs) and SDs were calculated where statistical differences were identified. Results: No differences at baseline in lower-extremity kinematics or EMG were found between groups. Following rehabilitation, the PENS group had significant reduction in hip adduction between $29 \%$ and $47 \%$ of the SLS (MD $=4.62^{\circ}\left[3.85^{\circ}\right]$ ) and between $43 \%$ and $69 \%$ of the SDT $\left(\mathrm{MD}=6.55^{\circ}\left[0.77^{\circ}\right]\right)$. Throughout the entire SDT, there was a decrease in trunk flexion in the PENS group $\left(\mathrm{MD}=10.91^{\circ}\left[1.73^{\circ}\right]\right)$. A significant decrease in gluteus medius activity was seen during both the SLS $(\mathrm{MD}=2.77$ [3.58]) and SDT (MD = 4.36 [5.38]), and gluteus maximus during the SLS (MD = 1.49 [1.46]). No differences were seen in the Sham group lower-extremity kinematics for either task. Conclusion: Rehabilitation with PENS improved kinematics in both tasks and decreased EMG activity. This suggests that rehabilitation with PENS may improve muscle function during functional tasks.
\end{abstract}

Keywords: anterior knee pain, functional tasks, movement patterns, muscle activity

Patellofemoral pain (PFP) is one of the most common knee pathologies seen in clinical practice. ${ }^{1,2}$ Females are more likely to have a history of PFP and have double the risk of developing PFP compared with their male counterparts. ${ }^{3}$ PFP patients present with retro- or peripatellar pain during a variety of functional tasks, such as squatting and stair ambulation. This pain has been linked to a decrease in activity level, ${ }^{4}$ decreased quality of life, ${ }^{5}$ and long-term consequences following the initial diagnosis. ${ }^{6,7}$

While the etiology is unknown, a multitude of neuromuscular impairments have been identified in patients suffering from PFP. Lower-extremity muscle weakness, faulty lower-extremity activation patterns, and altered movement patterns are common in females with PFP..$^{8-13}$ These factors are modifiable, yet researchers have not identified whether these impairments result in PFP or

Glaviano is with the School of Exercise and Rehabilitation Sciences, College of Health and Human Services, The University of Toledo, Toledo, OH, USA. Marshall is with the Department of Health \& Exercise Science, Appalachian State University, Boone, NC, USA. Mangum is with the College of Health Professionals and Sciences, University of Central Florida, Orlando, FL, USA. Hart, Hertel, and Saliba are with the Exercise and Sport Injury Laboratory, Department of Kinesiology, University of Virginia, Charlottesville, VA, USA. Hart and Russell are with the Department of Orthopedic Surgery, University of Virginia, Charlottesville, VA, USA. Glaviano (Neal.Glaviano@UToledo.edu) is corresponding author. are a consequence of PFP. Finding therapeutic interventions that specifically target these impairments, such as improving lowerextremity movement patterns and muscle activity during pain provoking tasks like squatting and stair ambulation, may improve overall outcomes in rehabilitation.

Females with PFP often present with lower-extremity muscle weakness, specifically their quadriceps and gluteus medius (GMed). ${ }^{10,14}$ Quadriceps atrophy is often seen in PFP patients, resulting in knee extension weakness. ${ }^{15,16}$ Weakness of the GMed is also often reported, as females with PFP have been found to be $26 \%$ weaker during hip abduction strength testing and $24 \%$ to $36 \%$ weaker in hip external rotation strength testing when compared with healthy controls. ${ }^{8,10}$ GMed is responsible for hip abduction and external rotation, and individuals with GMed weakness may be unable to eccentrically control these excursions during functional tasks. ${ }^{9}$ Strength deficits are commonly addressed during rehabilitation that often results in good short-term outcomes, but pain often returns within 3 months and remains for years. ${ }^{17}$

In addition to muscle weakness, groups of PFP patients also have altered lower-extremity muscle activity during a variety of functional tasks when measured by electromyography (EMG). ${ }^{8,11,18}$ Decreases in muscle activity in both the vastus medialis oblique (VMO) and GMed have been found during squatting and stair ambulation tasks. ${ }^{18,19}$ It has been suggested that these impairments 
alter patellar tracking and increase frontal plane movement during squatting and step-down tasks (SDTs), which may increase pressure of the patella on the trochlear groove during flexion-based tasks. ${ }^{19-21}$

Impairments in both strength and muscle activation have been suggested to influence aberrant movement patterns during functional tasks. ${ }^{12}$ Individuals with PFP present with increased hip adduction and internal rotation during weight-bearing tasks, which increase stress on the patellofemoral joint and may result in an increase in pain. ${ }^{22,23}$ Willson and Davis ${ }^{21}$ reported that as the demands of functional tasks increase so does the extent of hip adduction and internal rotation compared with healthy controls. Clinicians target these altered movement patterns by supplementing traditional rehabilitation with movement retraining. ${ }^{24-26}$ While relative hip abduction and external rotation are improved during the trained task, there is limited transfer to other pain provoking tasks. ${ }^{26}$

The incorporation of electrical stimulation is one potential intervention to reeducate altered neuromuscular function in individuals with PFP. While traditional neuromuscular electrical stimulation treatments produce tetanus contractions, novel forms such as patterned electrical neuromuscular stimulation (PENS) incorporate a more natural muscle contraction sequence and are more comfortable. PENS delivers a precisely timed stimulus to these muscles based off of healthy EMG firing patterns and is targeted to the agonist, briefly to the antagonist muscle and then to the agonist. ${ }^{27}$ PENS has been utilized to target both the GMed and VMO. An intervention utilizing a single PENS application on individuals with PFP has been found to increase GMed activation 2 -fold and decrease hip adduction for $32 \%$ of the task during a lateral step-down exercise. ${ }^{27,28}$

Previous studies evaluating traditional rehabilitation for PFP reported strength gains and improvements in patient-reported function. ${ }^{29,30}$ Despite these findings, PFP often recurs, and there are poor long-term outcomes. Traditional rehabilitation has not been shown to alter muscle activation or kinematics long term. Therefore, the purpose of this study was to determine the effect of PENS augmented rehabilitation on lower-extremity movement patterns and muscle activity during functional tasks in females with PFP.

\section{Methods}

A double-blinded, randomized controlled laboratory study (NCT02441712) was conducted to evaluate the influence of 4-week rehabilitation program with PENS on lower-extremity kinematics and EMG activity in females with PFP. This was part of a larger study that evaluated patient-reported outcomes, lower-extremity strength, and range of motion following the rehabilitation program. ${ }^{31}$ Dependent variables were lower-extremity and trunk kinematics (frontal and sagittal), and EMG activity of the lower-extremity (VMO, vastus lateralis [VL],GMed, gluteus maximus [GMax], biceps femoris [BF], and adductor longus [ADD]) throughout $100 \%$ of each task. Independent variables were time (prerehabilitation and postrehabilitation) and group (PENS and Sham).

\section{Participants}

Volunteers included a convenient sample of 16 females with PFP, recruited from a local university and community between June 2015 and April 2016 (Table 1 and Figure 1). A licensed athletic trainer evaluated patients to determine inclusion/exclusion criteria for study enrollment (Table 2), following recommendations from previous studies. ${ }^{27}$ Patients with bilateral knee pain self-defined
Table 1 Baseline Anthropometric, Subjective, and Objective Characteristics

\begin{tabular}{lccc}
\hline Demographics & PENS $(\mathbf{n}=\mathbf{8})$ & Sham $(\mathbf{n}=\mathbf{8})$ & $\boldsymbol{P}$ value \\
\hline Age, y & $23.0(6.0)$ & $23.5(4.0)$ & .85 \\
Height, cm & $166.8(5.7)$ & $165.3(6.4)$ & .62 \\
Mass, kg & $65.7(9.6)$ & $66.8(17.3)$ & .88 \\
Duration, mo & $28.0(30.6)$ & $24.5(31.3)$ & .82 \\
C-VAS & $2.2(2.1)$ & $1.5(0.7)$ & .12 \\
AKPS & $77.7(6.5)$ & $71.8(8.1)$ & .13 \\
\hline
\end{tabular}

Abbreviations: AKPS, anterior knee pain scale; C-VAS, current visual analog scale; PENS, patterned electrical neuromuscular stimulation.

their worse limb, which was used for both testing and rehabilitation program. All patients completed written consent prior to enrollment, and the University of Virginia Institutional Review Board approved the study.

\section{Procedures}

A single researcher completed all initial and final assessment measures (N.R.G.) and was blinded to group membership. All patients were then allowed to warm-up for 5 minutes of treadmill walking at a self-selected pace. Patients also completed a visual analog scale (VAS) to mark their current knee pain before testing. The VAS is a reliable and valid assessment for pain during functional tasks in individuals with PFP. ${ }^{32}$

Electromyography was collected on selected muscles simultaneously using the Trigno wireless EMG system (Delsys, Boston, MA) integrated to the Motion Monitor software (Ascension Technology, Chicago, IL). A $2000-\mathrm{Hz}$ sampling rate was selected, with a 10 - to $500-\mathrm{Hz}$ band-pass filter and 50-Hz notch filter. EMG sensors were placed over the BF, GMax, GMed, VL, VMO, and ADD, according to surface electromyography for the non-invasive assessment of muscles (SENIAM) recommendations. ${ }^{33}$ Electrode preparation included shaving, debriding, and isopropyl alcohol cleansing over the muscle belly of interest. EMG electrode placement was confirmed with quiet standing and maximal voluntary isometric contractions to minimize cross-talk and ensure appropriate placement.

Three-dimensional kinematics were collected with a 12-camera motion analysis system (VICON motion systems, Culver City, CA) in conjunction with Motion Monitor software (Ascension Technology). Rigid 4-cluster reflective marker sets were secured over the dorsum of each foot, lateral shanks, lateral thighs, the lumbar spine, and upper thorax. Rigid marker sets were secured with Velcro straps and self-adhesive tape. Kinematic data were collected at $250 \mathrm{~Hz}$. A skeleton model was created by digitizing the top of the subject's head, C7, T12, L5, bilateral ASIS, medial and lateral knee joints, medial and lateral malleolus, and second phalanx. All tasks were completed on a dual belt Bertec ${ }^{\mathrm{TM}}$ Fully Instrumented Treadmill (Bertec, Columbus, $\mathrm{OH}$ ).

Following EMG and motion analysis setup, a quiet standing trial was recorded for 5 seconds and served as normalization for EMG and for determining kinematic excursion during the functional tasks. Following setup, 2 functional assessments were conducted, a single-leg squat (SLS) and a SDT, which were conducted in this order for all patients at both testing time periods. Patients were provided verbal instructions to complete the task, and 3 practice trials of each were provided. For the SLS, patients stood on the limb of interest, arms across their chest, and nontest limb flexed to $90^{\circ}$. Patients were instructed to lower themselves as low 


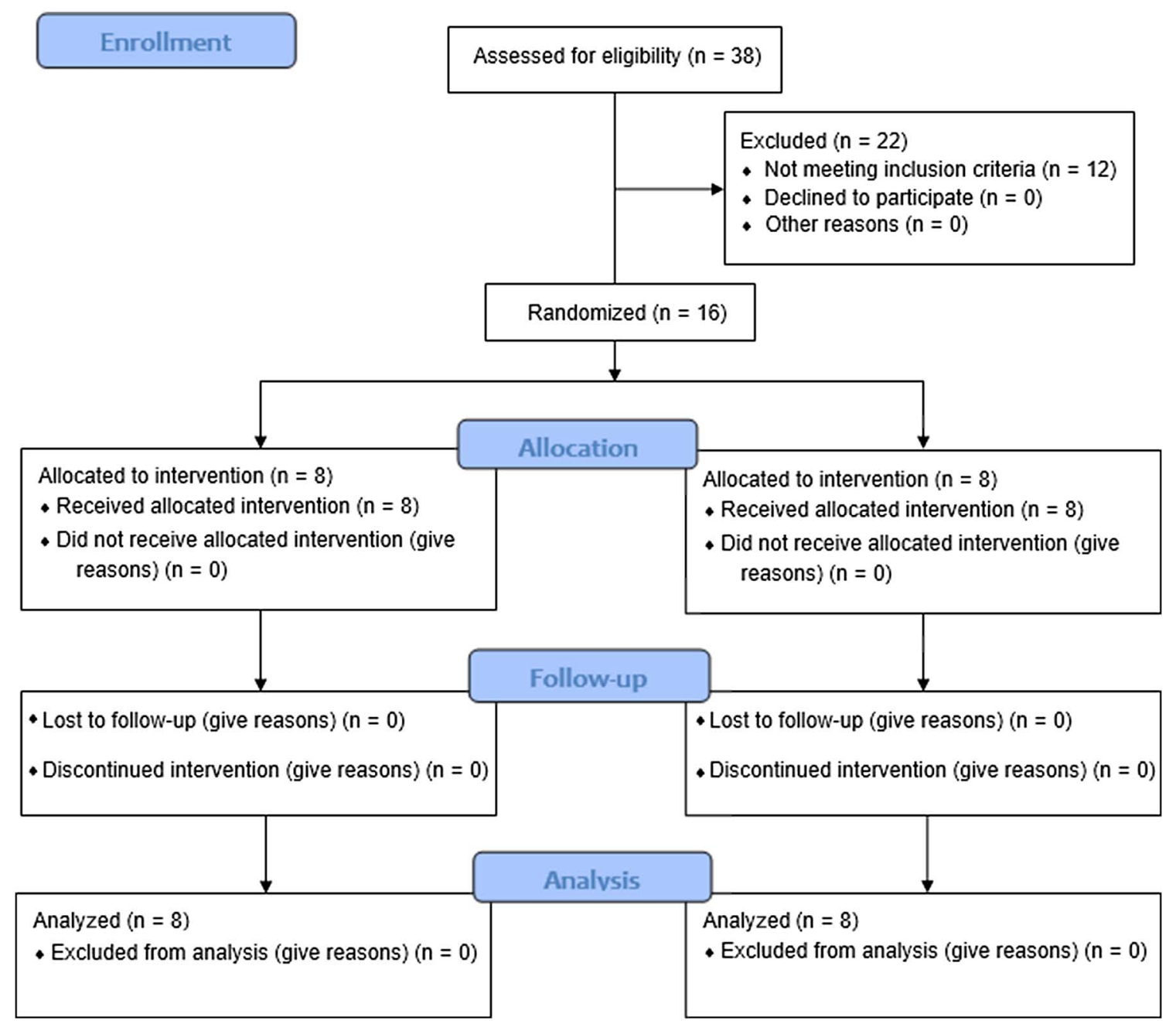

Figure 1 - CONSORT flowchart.

as possible for 2 seconds and return to a fully extended knee position for 2 seconds. ${ }^{27}$ Depth of squat was quantified in the motion analysis software, and if $60^{\circ}$ of knee flexion was not achieved, researchers provided verbal instructions to squat deeper. ${ }^{27}$ A metronome was utilized to provide auditory feedback for the duration of the task, standardizing the squat speed to be 4 seconds in total (2-s descend and 2-s ascend). ${ }^{27}$ The only cueing provided to patients was relative to the depth and speed of the squat. Three individual trials were conducted with a 30 -second rest period between each trial. Following the third squat, individuals were asked to assess their knee pain during the SLS with the VAS.

A 1-minute break was provided following the SLS. During this time, patients were provided instructions for the anterior SDT followed by 3 practice trials. A $21-\mathrm{cm}$ step was positioned next to a nonconductive force plate (Bertec). Patients stood on the step with their pathological limb and maintained their hands on their hips. Individuals lowered themselves until the heel of their contralateral limb came in contact with force plate and then raised themselves back to the starting position. Patients were instructed to just touch the force plate and not transfer their full weight. A metronome was used to standardize the speed of the SDT to be completed over 4 seconds (2-s descend and 2-s ascend). ${ }^{27}$ Patients were only provided cueing if they completed the task at the improper speed, flexed their contralateral limb during the task, or completed a full weight transfer when making contact with the force plate. Individuals completed 5 consecutive SDT tasks at a self-selected rate. A separate VAS was used to assess the knee pain during the SDT.

\section{Interventions}

Patterned electrical neuromuscular stimulation was administered with the Omnistim ${ }^{\circledR}$ Pro Electrotherapy System (Accelerated Care Plus, Reno, NV) in a short-seated position at the start of each rehabilitation session. PENS is an asymmetrical biphasic square waved stimulus with a 70-microsecond phase duration, $50-\mathrm{Hz}$ pulse frequency, and stimulus train of 200 milliseconds..$^{28}$ Agonist/ antagonist muscle groups were targeted based on manufacturer's recommendations. Channel A provided electrical stimulation to the GMed and VMO, while channel B stimulated the adductor and hamstring muscles groups. Alternating stimulation was provided for channel A for 200 milliseconds, followed by a stimulus of channel B for 200 milliseconds, and an additional stimulus to channel A for 120 milliseconds. A 40-millisecond stimulus overlap occurred between each alternating stimulus. Amplitude was adjusted so that patients who received the PENS treatment had a strong but tolerable motor treatment for 15 minutes. Those patients randomized into the Sham group received an identical setup with a 1-mA subsensory 


\section{Table 2 Inclusion and Exclusion Criteria}

\begin{tabular}{l} 
Inclusion \\
- $18-35$ y old \\
- Nontraumatic retro- or peripatellar symptoms \\
- Pain with 2 or more of the following activities: \\
O Stair ambulation \\
O Kneeling \\
O Squatting \\
O Jumping \\
O Prolonged sitting \\
O Running \\
O Quadriceps contraction \\
O Pressure to the patella \\
- Pain for a minimal duration of 3 mo \\
- Score <85 on the anterior knee pain scale \\
Exclusion \\
- Previous history of knee surgery \\
- Ligamentous instability \\
- Additional sources of anterior knee pain \\
- Back or lower-extremity injury within the last year \\
- Neurological involvement \\
- Contraindications to electrical stimulation \\
O Implanted biomechanical devices \\
O Hypersensitivity to electrical stimulation \\
O Muscular abnormalities \\
O Infection to lower-extremity \\
\hline
\end{tabular}

treatment, also for 15 minutes, prior to therapeutic exercise. Delivering a $1-\mathrm{mA}$ subsensory treatment allowed the device display screen to be visible, flashing lights, and buzzer at the completion of the 15 minutes, which is identical to those in the PENS group. ${ }^{27,34}$ Those allocated to the Sham group were instructed they were receiving a subsensory treatment. 27,34

Individuals completed a 4-week rehabilitation program in a laboratory setting ( 3 supervised visits a week) that incorporated quadriceps; gluteals; and core strengthening, stretching, and balance training, using an impairment-based paradigm (Table 3). ${ }^{35}$ This rehabilitation program is a modified version of a program that produced improvements in strength and subjective function in individuals with $\mathrm{PFP}^{30}$ and was individualized to each patient based on evaluation findings in each case. A single certified athletic trainer (A.N.M.) completed all electrical stimulation treatments and progression of all exercise throughout the duration of the study. Patients were continuously assessed on difficultly of each exercise and progressed throughout the 4 weeks based off of the clinical judgment of the athletic trainer to mimic clinical practice. This individual also assigned participants to treatment group. Randomization to the treatment allocation was done in a 4-block method (Excel; Microsoft, Redmond, WA) using concealed envelops by a member of the research team who did not collect data nor provide interventions (S.S.).

\section{Reassessment}

Patients returned to the laboratory within 96 hours of the completion of the final rehabilitation session, which was identical to baseline testing. Patients completed 3 SLS and 5 consecutive SDT with VAS scores for each task, for the postrehabilitation measures.

\section{Data Processing}

Kinematic data were filtered with a 6-Hz, fourth-order, zero-lag, low-pass Butterworth filter. Euler angles $(Y, X$, and $Z)$ were used to identify flexion/extension, adduction/abduction, and internal/ external rotation. Raw EMG data were filtered with a 50-ms root mean squared moving window and normalized to quiet standing EMG activity. Both kinematic and EMG data were reduced to 101 data points from the initiation of knee flexion to full knee extension for each task, to represent $0 \%$ to $100 \%$ of the task. ${ }^{36,37}$ Data were exported from Motion Monitor software, and an average of 3 trials was calculated for each task.

\section{Statistical Analysis}

To determine group differences (PENS vs Sham) during the SLS and SDT, curve analyses were constructed for lower-extremity kinematics and EMG activity across the tasks. Curve analyses were also constructed to compare lower-extremity kinematics and EMG activity prerehabilitation and postrehabilitation between groups. Group means were plotted for the duration of the tasks with $90 \%$ confidence intervals. $^{36,37}$ Significant differences were identified when the confidence intervals did not overlap for 3 consecutive data points between the 2 groups. ${ }^{36,37}$ Mean differences (MDs) and SDs were calculated during any phases of statistical significance. ${ }^{36,37}$ Independent $t$ tests were used to compare anthropometric data and pain during functional tasks between the groups, with alpha set a priori at $P<.05$. Excel (version 2016; Microsoft) was used to create curve analyses and calculate MD, while SPSS (version 20.0; SPSS, Inc, Chicago, IL) was used to compare anthropometric and pain data.

\section{Results}

No significant differences were identified between the PENS or Sham groups for any anthropometric variables (Table 1). At baseline, we found no significant differences in lower-extremity kinematic or EMG variables during the SLS (Supplemental Figures 1 and 2 [available online]) or SDT (Supplemental Figures 3 and 4 [available online]), or current pain between the PENS (2.20 [2.15]) and Sham (1.58 [0.68]) groups.

\section{Pain}

Current pain significantly decreased following the intervention in both the PENS (pre: 2.20 [2.15], post: 0.71 [0.61], $P=.04$ ) and Sham (pre: 1.58 [0.68], post: 0.68 [0.84], $P=.04$ ) groups. Pain during the functional tasks did not decrease significantly following the intervention in either the PENS group during the SLS (pre: 1.69 [1.56], post: 0.90 [1.07], $P=.26$ ) or SDT (pre: 1.19 [1.25], post: 0.78 [0.69], $P=.43$ ). Pain also did not decrease significantly in the Sham group during the SLS (pre: 2.60 [2.87], post: 0.90 [1.10], $P=.14$ ) or SDT (pre: 1.95 [1.79], post: 0.96 [0.83], $P=.18$ ).

\section{Frontal Plane Kinematics}

We found significant improvement in frontal plane hip kinematics for individuals allocated into the PENS treatment group. 
Table 3 Rehabilitation Program

\begin{tabular}{|c|c|c|c|c|c|}
\hline Weeks & Exercise & Set & $\begin{array}{l}\text { Repetitions } \\
\text { or seconds }\end{array}$ & Initial load & $\begin{array}{l}\text { Exercise progression } \\
\text { increase }\end{array}$ \\
\hline \multirow[t]{9}{*}{$1-2$} & 4-way SLR & 2 & 20 & $50 \% 1 \mathrm{RM}$ & $0.5 \mathrm{~kg}$ \\
\hline & Seated knee flexion and extension & 2 & 20 & $50 \% 1 \mathrm{RM}$ & $2-5 \mathrm{~kg}$ \\
\hline & Wall squats & 2 & 20 & 5-s hold & 2-s hold \\
\hline & Isometric hip $\mathrm{Abd} / \mathrm{ER}$ & 2 & 20 & 5-s hold & 2-s hold \\
\hline & Clam shells & 2 & 20 & 2 elastic-resistance levels below 1RM & Elastic-resistance level \\
\hline & Pelvic tilt prone & 2 & 15 & 10-s hold & $5 \mathrm{~s}$ \\
\hline & Pelvic tilt on Swiss ball & 1 & 5 & $20 \mathrm{~s}$ & $5 \mathrm{~s}$ \\
\hline & Single-leg balance (eyes open) & 3 & $30 \mathrm{~s}$ & - & Surface and perturbation \\
\hline & Single-leg balance (eyes closed) & 3 & $30 \mathrm{~s}$ & - & Surface and perturbation \\
\hline \multirow[t]{14}{*}{$3-4$} & 4-way SLR & 3 & 12 & $75 \% 1 \mathrm{RM}$ & $0.5 \mathrm{~kg}$ \\
\hline & Seated knee flexion and extension & 3 & 12 & $75 \% 1 \mathrm{RM}$ & $2-5 \mathrm{~kg}$ \\
\hline & Wall squats & 3 & 12 & $10 \% \mathrm{BM}$ & $5 \% \mathrm{BM}$ \\
\hline & Step ups/downs & 3 & 12 & $10 \% \mathrm{BM}$ & $5 \% \mathrm{BM}$ \\
\hline & Lateral rotation in $\mathrm{CKC}$ & 3 & 12 & 1 elastic-resistance level below $1 \mathrm{RM}$ & Elastic-resistance level \\
\hline & Pelvic drops & 3 & 12 & $75 \% 1 \mathrm{RM}$ & $1-2 \mathrm{~kg}$ \\
\hline & Clam shells & 3 & 12 & 1 elastic-resistance level below $1 \mathrm{RM}$ & Elastic-resistance level \\
\hline & Planks (anterior and lateral) & 1 & 5 & $30 \mathrm{~s}$ & $5 \mathrm{~s}$ \\
\hline & Trunk extension on Swiss ball & 3 & 12 & - & 2 repetitions \\
\hline & Single-leg balance (eyes open) & 3 & $30 \mathrm{~s}$ & - & Surface and perturbation \\
\hline & Single-leg balance (eyes closed) & 3 & $30 \mathrm{~s}$ & - & Surface and perturbation \\
\hline & Single-leg squat with mirror training & 3 & 12 & 1 elastic-resistance level below $1 \mathrm{RM}$ & Elastic-resistance level \\
\hline & Lunge with mirror training & 3 & 12 & 1 elastic-resistance level below $1 \mathrm{RM}$ & Elastic-resistance level \\
\hline & Single-leg dead lift with mirror training & 3 & 12 & 1 elastic-resistance level below $1 \mathrm{RM}$ & Elastic-resistance level \\
\hline
\end{tabular}

Abbreviations: 1RM, 1-repetition maximum; Abd, abduction; BM, body mass; CKC, closed kinetic chain; ER, external rotation; SLR, straight leg raise.

A decrease in hip adduction was seen between $29 \%$ and $47 \%$ $\left(\mathrm{MD}=4.62^{\circ}\left[3.85^{\circ}\right]\right)$ of the SLS task (Figure 2$)$ and $43 \%$ and $69 \%$ $\left(\mathrm{MD}=6.55^{\circ}\left[0.77^{\circ}\right]\right)$ of the SDT (Figure 3). No differences in trunk or knee frontal plane kinematics were seen in the PENS group. The Sham group did not produce any changes in knee, hip, or trunk kinematics for either the SLS or SDT.

\section{Sagittal Plane Kinematics}

Changes in sagittal kinematics were observed following 4 weeks of rehabilitation with PENS in each of the 2 tasks. A decrease in knee flexion was seen for $11 \%$ to $26 \%\left(\mathrm{MD}=6.76^{\circ}\left[6.09^{\circ}\right]\right), 66 \%$ to $81 \%\left(\mathrm{MD}=9.16^{\circ}\left[9.04^{\circ}\right]\right)$, and $86 \%$ to $100 \%\left(\mathrm{MD}=5.45^{\circ}\right.$ $\left[3.85^{\circ}\right]$ ) of the SLS (Figure 2). An increase in hip flexion was seen during the SDT for $10 \%$ to $42 \%\left(\mathrm{MD}=6.82^{\circ}\left[11.19^{\circ}\right]\right)$ and $94 \%$ to $100 \%\left(\mathrm{MD}=5.62^{\circ}\left[1.27^{\circ}\right]\right)$. Trunk flexion decreased across the entire SDT $\left(\mathrm{MD}=10.91^{\circ}\left[1.73^{\circ}\right]\right)$ (Figure 3). No differences in sagittal plane kinematics were seen in the Sham group for either task.

\section{EMG Activation}

Following the intervention, the PENS group had a decrease in VL activation during $57 \%$ to $63 \%$ of the SLS (MD $=7.42 \%$ [10.40\%]) (Figure 4). The PENS group experienced a decrease in GMax activation between $4 \%$ and $47 \%$ of the SLS (MD $=1.49 \%$ [1.46\%]) and decrease in GMed between 39\% and 77\% (MD $=2.77 \%$ [3.58\%]) (Figure 4). Adductor activation was decreased during the SLS, with significant differences between 1\% and 19\%
$(\mathrm{MD}=0.98 \%[1.01 \%]), 22 \%$ and $54 \%(\mathrm{MD}=2.66 \%[2.36 \%])$, $61 \%$ and $65 \%(\mathrm{MD}=3.69 \%[5.06 \%])$, and $87 \%$ and $100 \%(\mathrm{MD}=$ $1.88 \%$ [1.69\%]) (Figure 4). BF activity was also reduced between $11 \%$ and $71 \%(\mathrm{MD}=2.65 \%$ [2.47\%] $)$ and $90 \%$ and $100 \%(\mathrm{MD}=$ $2.47 \%$ [2.51\%]) (Figure 4). The Sham group demonstrated an increase in VL activity between $49 \%$ and $62 \%(\mathrm{MD}=-17.10 \%$ [19.70\%]) and a decrease in ADD activity between $58 \%$ and $65 \%$ $(\mathrm{MD}=5.08 \%[7.34 \%])$. No other significant differences were seen in the Sham group during the SLS.

There were no differences in the VMO or VL EMG activity following the intervention during the SDT for either the PENS or Sham group. Those in the PENS group had a reduction in GMax activity during $4 \%$ to $13 \%$ ( $\mathrm{MD}=1.21 \%$ [1.41\%]), $17 \%$ to $46 \%(\mathrm{MD}=1.03 \%[1.09 \%])$, and $67 \%$ to $91 \%(\mathrm{MD}=3.74 \%$ [3.53\%]), and GMed between $72 \%$ and $80 \%$ (MD $=4.36 \%$ [5.38\%]) (Figure 5). ADD activity also decreased during $1 \%$ to $10 \%$ (MD $=0.97 \%[1.07 \%]$ ) of the SDT as well as BF during $1 \%$ to $60 \%(\mathrm{MD}=3.35 \%$ [3.04\%]) and $81 \%$ to $100 \%(\mathrm{MD}=3.03 \%$ [2.78\%]) (Figure 5). No differences in SDT EMG activity were seen in the Sham group following the intervention.

\section{Discussion}

The purpose of this study was to evaluate the effects of an impairment-based rehabilitation program augmented with either a PENS or Sham treatment on lower-extremity kinematics or muscle activity in females with PFP. We identified improvements in both frontal and sagittal plane hip, knee, and trunk kinematics in 

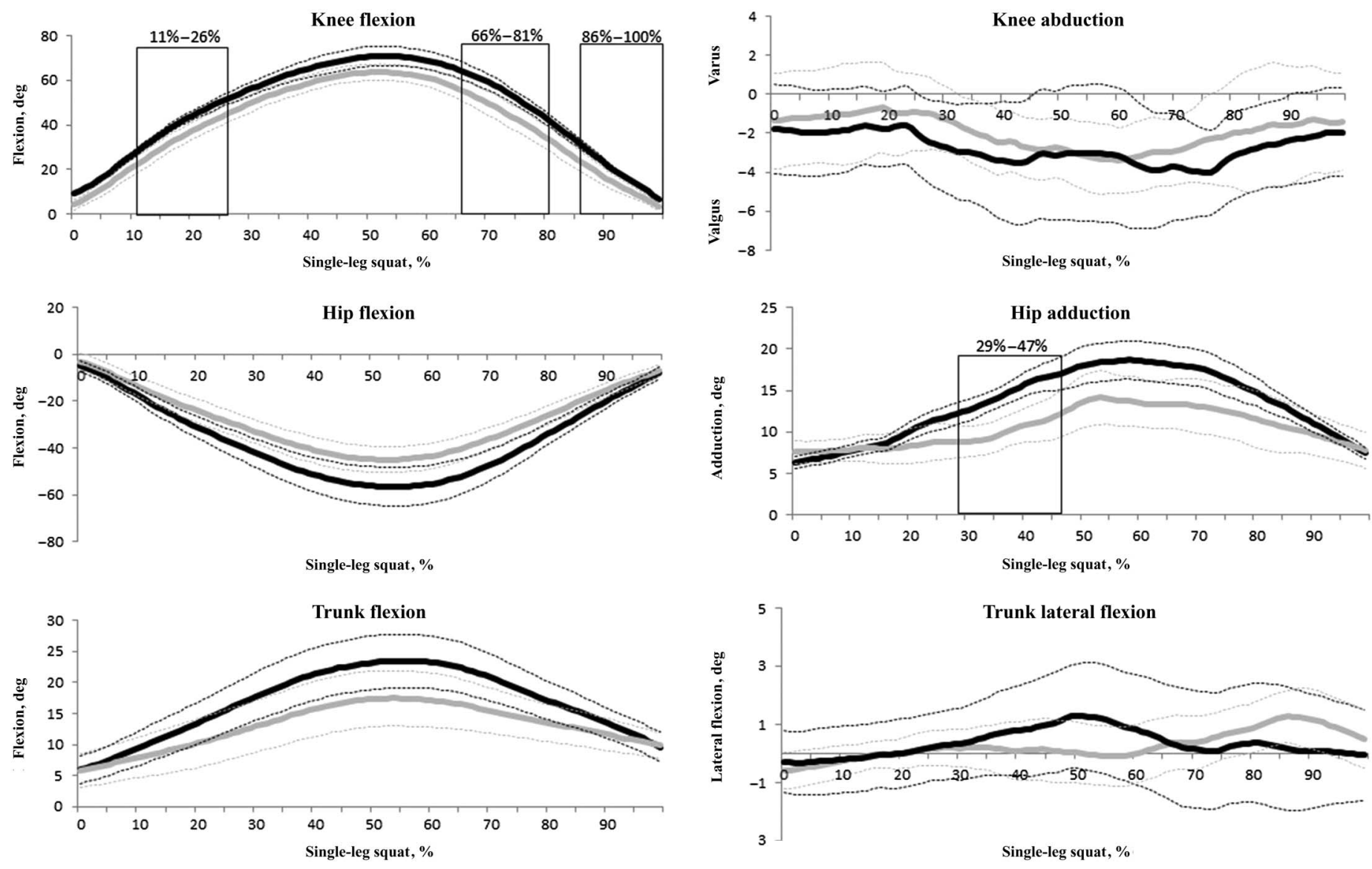

Figure 2 - Differences in lower-extremity kinematics during the single-leg squat at preintervention (black lines) and postintervention (gray lines) for those in the patterned electrical neuromuscular stimulation group. Solid lines represent mean group values and dotted lines represent the upper and lower $90 \%$ confidence intervals. Statistical differences are boxed, with the exact percentage of the squatting task where differences were identified being provided above the box.

individuals who received rehabilitation with PENS during both a SLS and SDT. We also found a decrease in muscle activity of the lower-extremity, including a decrease in GMed, GMax, ADD, and BF during both functional tasks when PENS was administered prior to each of the 12 supervised rehabilitation sessions. A significant decrease in VL activity was only seen during the SDT in the PENS group.

Hip adduction was reduced across the task following rehabilitation with PENS (Figures 2 and 3). While strength training has not been consistently beneficial, real-time retraining programs have been another avenue to improve altered movement patterns. Noehren et $\mathrm{al}^{26}$ conducted real-time gait retraining for 2 weeks with rehabilitation and found improvement in hip adduction and internal rotation while running. The authors observed a decrease of $5^{\circ}, 26$ which is similar to our reduction of $6^{\circ}$ in both tasks. However, their improvements were only seen within the trained task (ie, gait) and did not transfer to a SLS. Willy and Davis ${ }^{24}$ saw similar results, as mirror training with the SLS improved squatting mechanics, but did not alter running mechanics. While not evaluated in this study, finding treatment options that transfer to multiple pain provoking activities may be one method to decrease the long-term presentation of PFP symptoms and improve the quality of life for these individuals.

Recent attention has been placed on the role of the trunk during functional tasks in individuals with PFP. ${ }^{9,38}$ Females have been found to have increased trunk excursions during tasks like the SLS, but this has not been evaluated consistently across different tasks. ${ }^{9}$ We found similar amounts of trunk flexion between groups during the SDT task. However, patients in the PENS group reduced their trunk flexion by over $10^{\circ}$ across the duration of the SDT postrehabilitation (Figure 3). Increased trunk flexion has been suggested as a protective mechanism in individuals who present with lower-extremity weakness. ${ }^{38,39}$ If quadriceps weakness is present, an increase in trunk flexion decreases the external moment needed by the quadriceps to complete the task..$^{39}$ This adaptation may also play a role in reducing pain, as a decrease in quadriceps force may be accompanied by changes in patellofemoral joint stress. ${ }^{39}$ While we did not evaluate the relationships between changes in quadriceps strength and trunk kinematics, there may be a potential relationship present in strength gains and improvements in trunk movement patterns. Additional attention should be evaluated on the role of muscle strength gains and trunk kinematics during functional tasks.

Clinicians have placed a great deal of focus on therapeutic exercises to address deficits in strength, muscle activity, and movement patterns in patients with PFP. Strength-focused rehabilitation has been found to improve lower-extremity strength, decrease pain, and improve subjective function. ${ }^{18,29,40}$ However, GMed strengthening programs have not altered muscle activity of the GMed during stair tasks. ${ }^{18}$ Traditional rehabilitation also did 

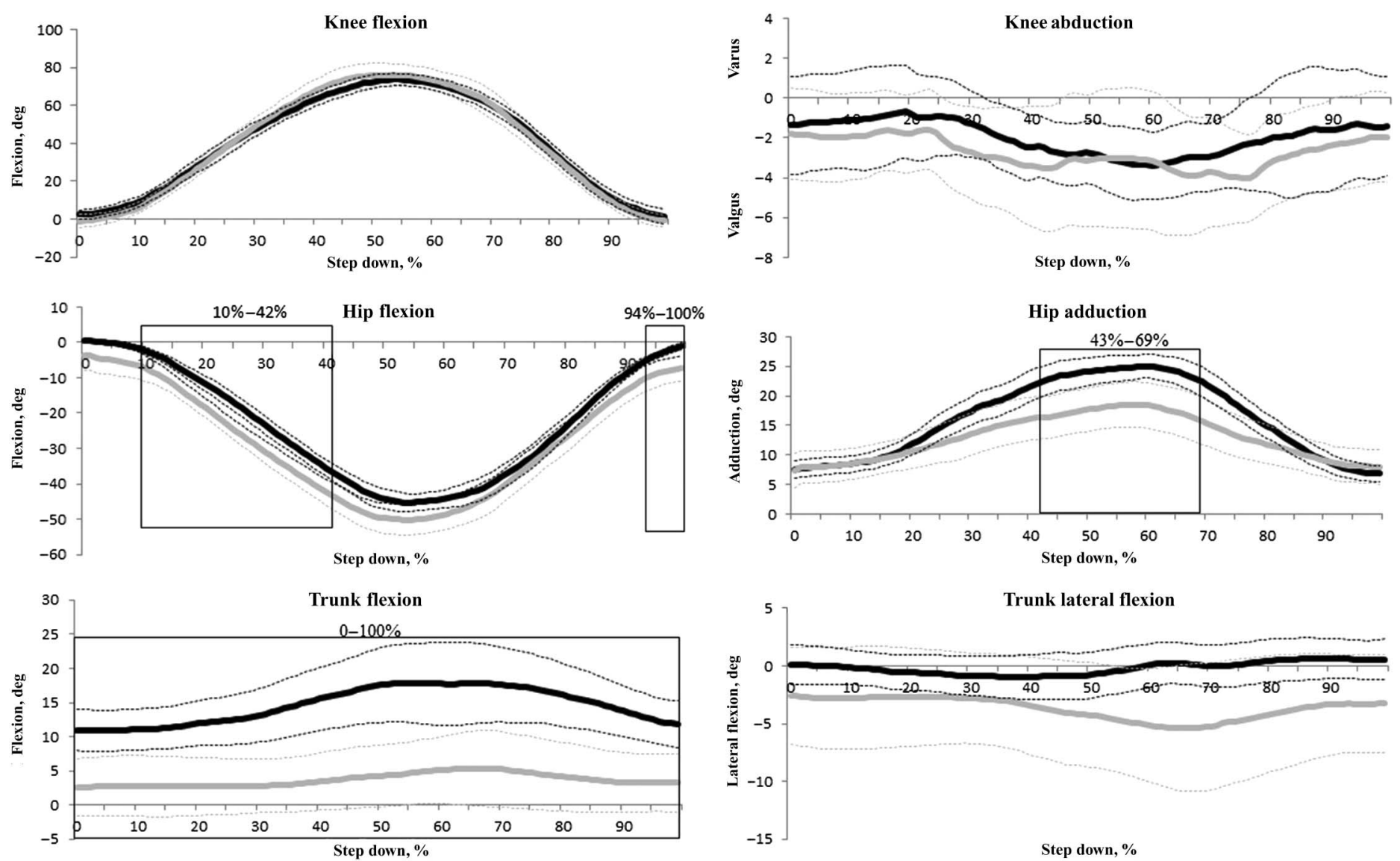

Figure 3 - Differences in lower-extremity kinematics during the step-down task at preintervention (black lines) and postintervention (gray lines) for those in the patterned electrical neuromuscular stimulation group. Solid lines represent mean group values and dotted lines represent the upper and lower $90 \%$ confidence intervals. Statistical differences are boxed, with the exact percentage of the step-down task where differences were identified being provided above the box.

not improve the altered movement patterns in those with PFP. We found similar findings in our Sham group, who completed a strength-focused intervention without changes to their lowerextremity kinematics or GMed activity during both tasks. Patients with PFP experience pain and dysfunction across numerous different tasks; therefore, improvements following rehabilitation must have the ability to be transferred across multiple tasks. Traditional rehabilitation, including GMed strengthening programs, does not typically improve EMG activation during SLS or SDT, suggesting additional interventions may be required to address these impairments. ${ }^{18}$

Both groups presented with similar baseline EMG activity across both tasks. While those in the Sham group did have small changes in the VL (49\%-62\%) and ADD (58\%-65\%) of the SLS, the majority of changes in lower-extremity activation was seen in the PENS group. This data come from a larger study that also evaluated subjective function and lower-extremity strength between the 2 groups (PENS and Sham) preintervention and postintervention. ${ }^{31}$ Both groups did improve in their subjective function and strength in that study; however, there was no difference between those in the PENS or Sham groups following the intervention. ${ }^{31} \mathrm{We}$ also did not see any differences in pain during the SLS or SDT in either group, limiting the possibility that a change in pain could explain the differences in EMG or kinematics. While there was no change in lower-extremity strength ${ }^{31}$ or pain between the groups, there were noted changes in lower-extremity kinematics, mainly in the PENS group. The decrease in frontal and sagittal plane kinematics could be due to improvements in neuromuscular control of the lower-extremity and a shift from a trunk pattern. The decrease in muscle activity may be related to changes in the neural drive, which may require less motor unit activation to be recruited to complete tasks like squatting and stair use. While there were improvements in the kinematics for both tasks, the increase in efficiency of the muscular contraction may be due to the underlying neural reeducation with the precisely timed recruitment of the GMed and VMO from the PENS treatment.

Previous studies are conflicting on changes in muscular activity following rehabilitation-based protocols. In those studies that describe improvements, ${ }^{41,42}$ the participants are often healthy individuals, and the strength training protocol is much longer, lasting over 20 weeks. While we saw a decrease in lower-extremity muscle activity after only 4 weeks of treatment, this conflicts with previous literature which has not identified changes in quadriceps or GMed muscle activity in other pathological populations. ${ }^{18,43} \mathrm{~A}$ proposed suggestion for the inconsistency in neural changes may be due to the population being assessed, as individuals with PFP have been found to have lower-extremity muscle inhibition. ${ }^{44}$ This decrease in motor unit availability may influence the recruitment of EMG activity during functional tasks; however, this relationship has not been evaluated to date. In addition, future research should 

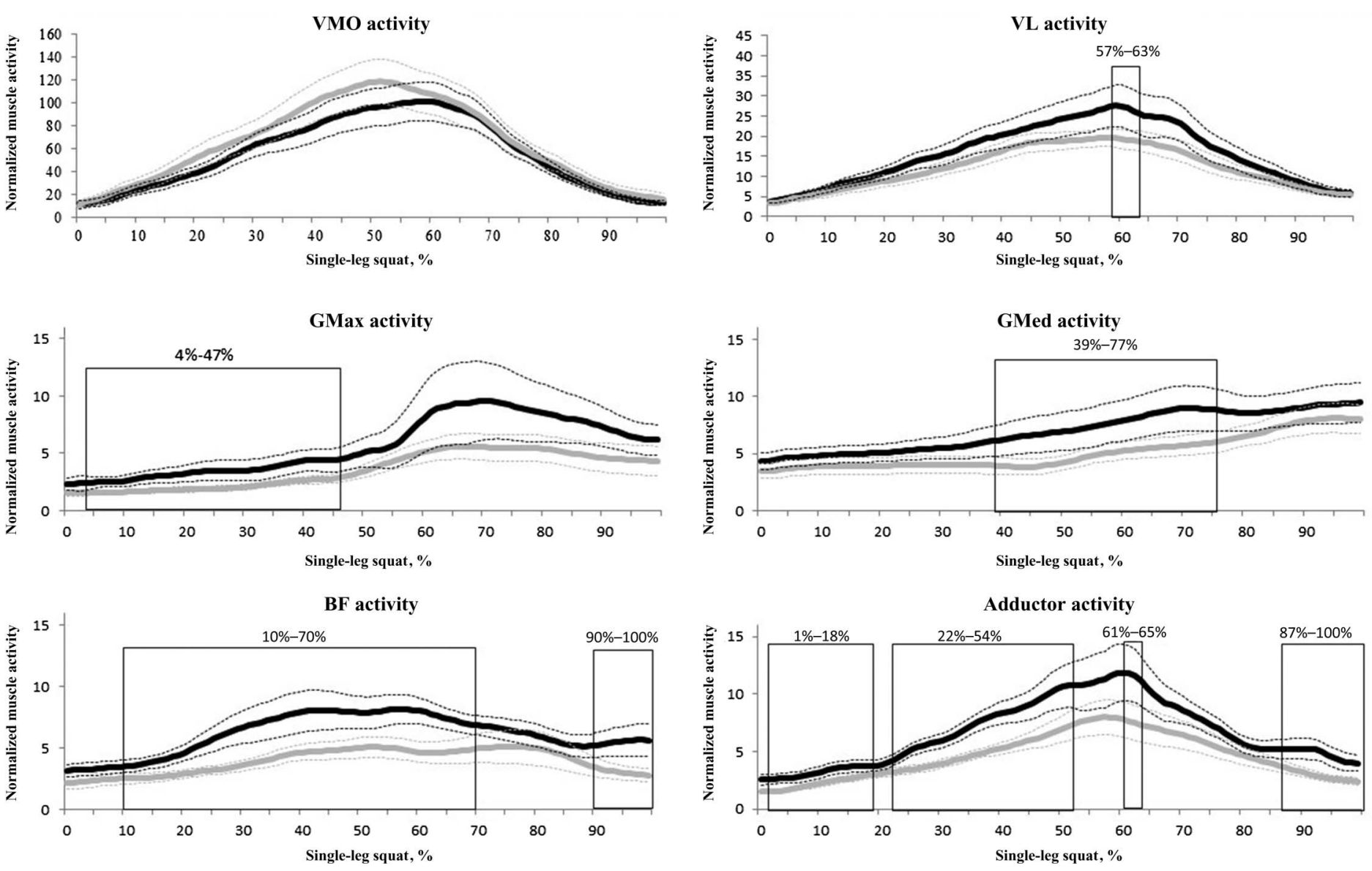

Figure 4 - Differences in muscle activation during the single-leg squat at preintervention (black lines) and postintervention (gray lines) for those in the patterned electrical neuromuscular stimulation group. Solid lines represent mean group values, and dotted lines represent the upper and lower $90 \%$ confidence intervals. Statistical differences are boxed, with the exact percentage of the squatting task where differences were identified being provided above the box. BF indicates biceps femoris; GMax, gluteus maximus; GMed, gluteus medius; VL, vastus lateralis; VMO, vastus medialis oblique.

evaluate more neural focused outcome measures following the application of PENS to determine if this novel form of electrical stimulation has an effect on neural function.

We did observe a significant decrease in both gluteal and adductor muscle activity during both tasks. While improvements in GMed and GMax activity may provide improved frontal plane control and minimize hip adduction, changes in adductor activity can also be attributed to the kinematic changes that are occurring. Previous authors have found that adductor activity is altered within the PFP population, ${ }^{45}$ as the 2 muscles work in opposition to control frontal plane motion. Although we compared the muscles independently, there is some evidence that a ratio between the muscles may provide insight into frontal kinematics within this population. ${ }^{46}$ Interestingly, the majority of the gluteal muscle activity changes in this study occurred during the middle phase of the tasks. The largest hip adduction excursions occur during this phase, thus requiring the greatest muscular control to provide stability to the hip and pelvis to complete the task.

Patterned electrical neuromuscular stimulation has been previously found to improve GMed activation and decrease hip adduction during a lateral SDT in individuals with PFP following a single application. ${ }^{27,28}$ There was also an immediate increase in the duration of activation in the GMed during the same task. ${ }^{27}$ However, no differences were seen in either muscle activity or kinematics during a SLS task, following one application of the treatment. On the contrary, we saw that 4 weeks of rehabilitation with PENS did produce improvement in both SLS and SDT, suggesting that long-term application of the modality in conjunction with an appropriate rehabilitation protocol may be needed to improve altered movement patterns across multiple tasks.

There are some limitations in this study. We conducted a RCT with a relatively small sample size, which may decrease the generalizability of our findings. Due to the heterogeneous presentation of impairments of individuals with PFP, a larger small sample size would provide additional insight into the effectiveness of this modality. Our patients also had substantial altered frontal plane movement during the functional tasks, and a large sample size would allow potential subanalyses depending on severity of the task. In addition, we did not randomize the order of the 2 functional tasks, which might influence our findings due to pain experienced by patients when completing these tasks. 

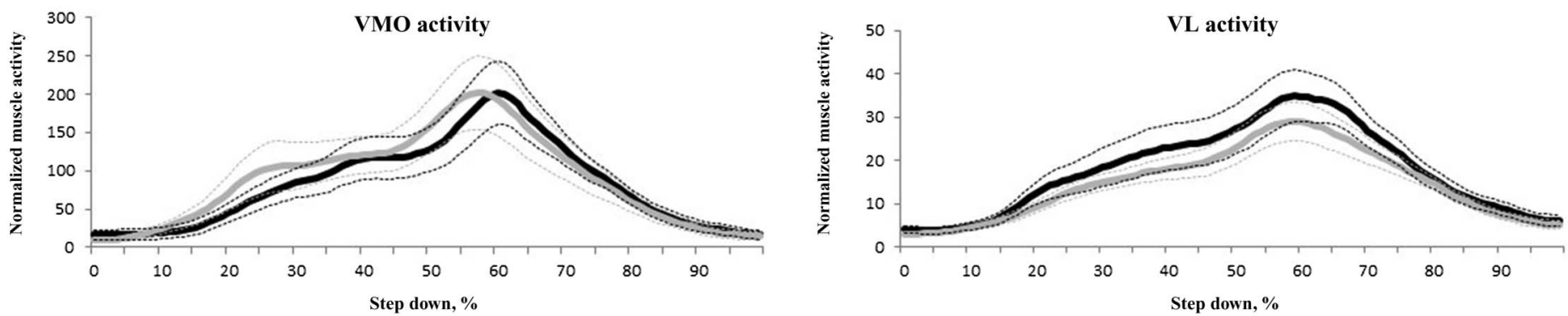

GMax activity
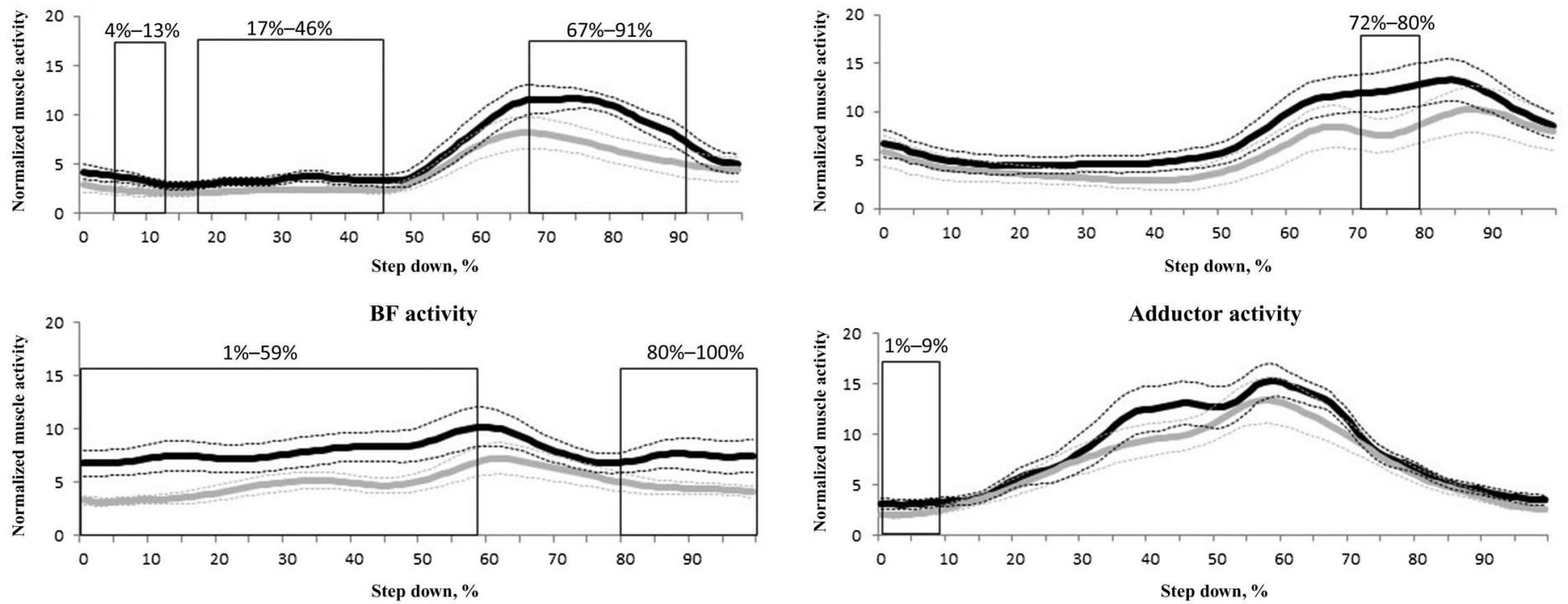

Figure 5 - Differences in muscle activation during the step-down task at preintervention (black lines) and postintervention (gray lines) for those in the patterned electrical neuromuscular stimulation group. Solid lines represent mean group values, and dotted lines represent the upper and lower $90 \%$ confidence intervals. Statistical differences are boxed, with the exact percentage of the step-down task where differences were identified being provided above the box. BF indicates biceps femoris; GMax, gluteus maximus; GMed, gluteus medius; VL, vastus lateralis; VMO, vastus medialis oblique.

\section{Conclusion}

Significant differences were found in lower-extremity kinematics and muscle activity in individuals who received rehabilitation with PENS. Utilization of PENS in conjunction with impairment-based rehabilitation may be helpful for clinicians who commonly treat PFP. These findings suggest a link potentially exists between changes in muscle activity following a rehabilitation program with PENS and lower-extremity kinematics during common pain provoking activities.

\section{Acknowledgments}

This study was funded by the Mid-Atlantic Athletic Trainers' Association and Accelerated Care Plus. Clinical Trial Registration: ClinicalTrials.gov. Registration study number: NCT02441712.

\section{References}

1. Glaviano NR, Kew M, Hart JM, Saliba S. Demographic and epidemiological trends in patellofemoral pain. Int J Sports Phys Ther. 2015;10(3):281-290. PubMed ID: 26075143
2. Taunton JE, Ryan MB, Clement DB, McKenzie DC, Lloyd-Smith DR, Zumbo BD. A retrospective case-control analysis of 2002 running injuries. Br J Sports Med. 2002;36(2):95-101. PubMed ID: 11916889 doi:10.1136/bjsm.36.2.95

3. Boling M, Padua D, Marshall S, Guskiewicz K, Pyne S, Beutler A. Gender differences in the incidence and prevalence of patellofemoral pain syndrome. Scand J Med Sci Sports. 2010;20(5):725-730. PubMed ID: 19765240 doi:10.1111/j.1600-0838.2009.00996.x

4. Glaviano NR, Baellow A, Saliba S. Physical activity levels in individuals with and without patellofemoral pain. Phys Ther Sport. 2017;27: 12-16. PubMed ID: 28780340 doi:10.1016/j.ptsp.2017.07.002

5. Cheung RT, Zhang Z, Ngai SP. Different relationships between the level of patellofemoral pain and quality of life in professional and amateur athletes. PM R. 2013;5(7):568-572. PubMed ID: 23375635 doi:10.1016/j.pmrj.2012.12.007

6. Stathopulu E, Baildam E. Anterior knee pain: a long-term follow-up. Rheumatology. 2003;42(2):380-382. PubMed ID: 12595641 doi:10. 1093/rheumatology/keg093

7. Rathleff MS, Roos EM, Olesen JL, Rasmussen S. Exercise during school hours when added to patient education improves outcome for 2 years in adolescent patellofemoral pain: a cluster randomised trial. Br J Sports Med. 2015;49(6):406-412. PubMed ID: 25388552 doi:10.1136/bjsports-2014-093929 
8. Ireland ML, Willson JD, Ballantyne BT, Davis IM. Hip strength in females with and without patellofemoral pain. J Orthop Sports Phys Ther. 2003;33(11):671-676. PubMed ID: 14669962 doi:10.2519/ jospt.2003.33.11.671

9. Nakagawa TH, Moriya ET, Maciel CD, Serrao FV. Trunk, pelvis, hip, and knee kinematics, hip strength, and gluteal muscle activation during a single-leg squat in males and females with and without patellofemoral pain syndrome. J Orthop Sports Phys Ther. 2012; 42(6):491-501. PubMed ID: 22402604 doi:10.2519/jospt.2012.3987

10. Robinson RL, Nee RJ. Analysis of hip strength in females seeking physical therapy treatment for unilateral patellofemoral pain syndrome. J Orthop Sports Phys Ther. 2007;37(5):232-238. PubMed ID: 17549951 doi:10.2519/jospt.2007.2439

11. Chester R, Smith TO, Sweeting D, Dixon J, Wood S, Song F. The relative timing of $\mathrm{VMO}$ and $\mathrm{VL}$ in the aetiology of anterior knee pain: a systematic review and meta-analysis. BMC Musculoskelet Disord. 2008;9:64. PubMed ID: 18452611 doi:10.1186/1471-2474-9-64

12. Barton CJ, Lack S, Malliaras P, Morrissey D. Gluteal muscle activity and patellofemoral pain syndrome: a systematic review. Br J Sports Med. 2013;47(4):207-214. PubMed ID: 22945929 doi:10.1136/ bjsports-2012-090953

13. Barton CJ, Levinger P, Menz HB, Webster KE. Kinematic gait characteristics associated with patellofemoral pain syndrome: a systematic review. Gait Posture. 2009;30(4):405-416. PubMed ID: 19651515 doi:10.1016/j.gaitpost.2009.07.109

14. Bolgla LA, Malone TR, Umberger BR, Uhl TL. Comparison of hip and knee strength and neuromuscular activity in subjects with and without patellofemoral pain syndrome. Int J Sports Phys Ther. 2011; 6(4):285-296. PubMed ID: 22163090

15. Giles LS, Webster KE, McClelland JA, Cook J. Does quadriceps atrophy exist in individuals with patellofemoral pain? A systematic literature review with meta-analysis. J Orthop Sports Phys Ther. 2013;43(11):766-776. PubMed ID: 24175596 doi:10.2519/jospt. 2013.4833

16. Kaya D, Citaker S, Kerimoglu U, et al. Women with patellofemoral pain syndrome have quadriceps femoris volume and strength deficiency. Knee Surg Sports Traumatol Arthrosc. 2011;19(2):242-247. PubMed ID: 20953760 doi:10.1007/s00167-010-1290-2

17. Lankhorst NE, van Middelkoop M, Crossley KM, et al. Factors that predict a poor outcome 5-8 years after the diagnosis of patellofemoral pain: a multicentre observational analysis. Br J Sports Med. 2015; 50(14):881-886. PubMed ID: 26463119 doi:10.1136/bjsports-2015094664

18. Boling MC, Bolgla LA, Mattacola CG, Uhl TL, Hosey RG. Outcomes of a weight-bearing rehabilitation program for patients diagnosed with patellofemoral pain syndrome. Arch Phys Med Rehabil. 2006; 87(11):1428-1435. PubMed ID: 17084115 doi:10.1016/j.apmr.2006. 07.264

19. Earl JE, Hertel J, Denegar CR. Patterns of dynamic malalignment, muscle activation, joint motion, and patellofemoral-pain syndrome. J Sport Rehabil. 2005;14:215-233.

20. Cowan SM, Bennell KL, Hodges PW, Crossley KM, McConnell J. Delayed onset of electromyographic activity of vastus medialis obliquus relative to vastus lateralis in subjects with patellofemoral pain syndrome. Arch Phys Med Rehabil. 2001;82(2):183-189. PubMed ID: 11239308 doi:10.1053/apmr.2001.19022

21. Willson JD, Davis IS. Lower extremity mechanics of females with and without patellofemoral pain across activities with progressively greater task demands. Clin Biomech. 2008;23(2):203-211. doi:10. 1016/j.clinbiomech.2007.08.025

22. Souza RB, Powers CM. Differences in hip kinematics, muscle strength, and muscle activation between subjects with and without patellofemoral pain. J Orthop Sports Phys Ther. 2009;39(1):12-19. PubMed ID: 19131677 doi:10.2519/jospt.2009.2885

23. Prins MR, van der Wurff P. Females with patellofemoral pain syndrome have weak hip muscles: a systematic review. Aust J Physiother. 2009;55(1):9-15. PubMed ID: 19226237 doi:10.1016/ S0004-9514(09)70055-8

24. Willy RW, Davis IS. The effect of a hip-strengthening program on mechanics during running and during a single-leg squat. J Orthop Sports Phys Ther. 2011;41(9):625-632. PubMed ID: 21765220 doi:10.2519/jospt.2011.3470

25. Willy RW, Davis IS. Varied response to mirror gait retraining of gluteus medius control, hip kinematics, pain, and function in 2 female runners with patellofemoral pain. J Orthop Sports Phys Ther. 2013;43(12):864-874. PubMed ID: 24175611 doi:10.2519/jospt. 2013.4516

26. Noehren B, Scholz J, Davis I. The effect of real-time gait retraining on hip kinematics, pain and function in subjects with patellofemoral pain syndrome. Br J Sports Med. 2011;45(9):691-696. PubMed ID: 20584755 doi:10.1136/bjsm.2009.069112

27. Glaviano NR, Huntsman S, Dembeck A, Hart JM, Saliba S. Improvements in kinematics, muscle activity and pain during functional tasks in females with patellofemoral pain following a single patterned electrical stimulation treatment. Clin Biomech. 2015;32: 20-27. doi:10.1016/j.clinbiomech.2015.12.007

28. Glaviano NR, Saliba SA. Immediate effect of patterned electrical neuromuscular stimulation on pain and muscle activation in individuals with patellofemoral pain. J Athl Train. 2016;51(2):118-128. PubMed ID: 26967547 doi:10.4085/1062-6050-51.4.06

29. Ferber R, Bolgla L, Earl-Boehm JE, Emery C, Hamstra-Wright K. Strengthening of the hip and core versus knee muscles for the treatment of patellofemoral pain: a multicenter randomized controlled trial. J Athl Train. 2015;50(4):366-377. PubMed ID: 25365133 doi:10.4085/1062-6050-49.3.70

30. Hamstra-Wright KL, Aydemir B, Earl-Boehm J, Bolgla L, Emery C, Ferber R. Lasting improvement of patient-reported outcomes 6 months after patellofemoral pain rehabilitation. J Sport Rehabil. 2017;26(4):223-233. PubMed ID: 27632841 doi:10.1123/jsr.20150176

31. Glaviano NR, Marshall AN, Mangum LC, et al. Impairment based rehabilitation with patterned electrical neuromuscular stimulation improves lower extremity function in individuals with patellofemoral pain: a preliminary study. J Athl Train. 2019;54(3):255-269. PubMed ID: 30721093 doi:10.4085/1062-6050-490-17

32. Crossley KM, Bennell KL, Cowan SM, Green S. Analysis of outcome measures for persons with patellofemoral pain: which are reliable and valid? Arch Phys Med Rehabil. 2004;85(5):815-822. PubMed ID: 15129407 doi:10.1016/S0003-9993(03)00613-0

33. Hermens HJ, Freriks B, Disselhorst-Klug C, Rau G. Development of recommendations for SEMG sensors and sensor placement procedures. J Electromyogr Kinesiol. 2000;10(5):361-374. PubMed ID: 11018445 doi:10.1016/S1050-6411(00)00027-4

34. Glaviano NR, Langston WT, Hart JM, Saliba S. Influence of patterned electrical neuromuscular stimulation on quadriceps activation in individuals with knee joint injury. Int J Sports Phys Ther. 2014;9(7):915-923. PubMed ID: 25540707

35. Glaviano NR, Saliba S. Impairment based rehabilitation for patellofemoral pain patients. Phys Sportsmed. 2016;44(3):311-323. PubMed ID: 27311015 doi:10.1080/00913847.2016.1200443

36. Chang M, Slater LV, Corbett RO, Hart JM, Hertel J. Muscle activation patterns of the lumbo-pelvic-hip complex during walking gait before and after exercise. Gait Posture. 2017;52:15-21. PubMed ID: 27846435 doi:10.1016/j.gaitpost.2016.11.016 
37. Chinn L, Dicharry J, Hart JM, Saliba S, Wilder R, Hertel J. Gait kinematics after taping in participants with chronic ankle instability. J Athl Train. 2014;49(3):322-330. PubMed ID: 24840583 doi:10. 4085/1062-6050-49.3.08

38. Schwane BG, Goerger BM, Goto S, Blackburn JT, Aguilar AJ, Padua DA. Trunk and lower extremity kinematics during stair descent in women with or without patellofemoral pain. J Athl Train. 2015; 50(7):704-712. PubMed ID: 25898109 doi:10.4085/1062-6050-49. 3.100

39. Powers CM. The influence of abnormal hip mechanics on knee injury: a biomechanical perspective. J Orthop Sports Phys Ther. 2010;40(2): 42-51. PubMed ID: 20118526 doi:10.2519/jospt.2010.3337

40. Earl JE, Hoch AZ. A proximal strengthening program improves pain, function, and biomechanics in women with patellofemoral pain syndrome. Am J Sports Med. 2011;39(1):154-163. PubMed ID: 20929936 doi:10.1177/0363546510379967

41. Hakkinen K, Alen M, Kallinen M, Newton RU, Kraemer WJ. Neuromuscular adaptation during prolonged strength training, detraining and re-strength-training in middle-aged and elderly people. Eur J Appl Physiol. 2000;83(1):51-62. PubMed ID: 11072774 doi:10.1007/s004210000248
42. Hakkinen K, Komi PV. Electromyographic changes during strength training and detraining. Med Sci Sports Exerc. 1983;15(6):455460. PubMed ID: 6656553 doi:10.1249/00005768-19831506000003

43. Wallerstein LF, Tricoli V, Barroso R, et al. Effects of strength and power training on neuromuscular variables in older adults. J Aging Phys Act. 2012;20(2):171-185. PubMed ID: 22472578 doi:10.1123/ japa.20.2.171

44. Hart JM, Pietrosimone B, Hertel J, Ingersoll CD. Quadriceps activation following knee injuries: a systematic review. J Athl Train. 2010;45(1):87-97. PubMed ID: 20064053 doi:10.4085/1062-605045.1.87

45. Aminaka N, Pietrosimone BG, Armstrong CW, Meszaros A, Gribble PA. Patellofemoral pain syndrome alters neuromuscular control and kinetics during stair ambulation. J Electromyogr Kinesiol. 2011; 21(4):645-651. PubMed ID: 21524921 doi:10.1016/j.jelekin.2011. 03.007

46. Mauntel TC, Begalle RL, Cram TR, et al. The effects of lower extremity muscle activation and passive range of motion on single leg squat performance. J Strength Cond Res. 2013;27(7):1813-1823. PubMed ID: 23096063 doi:10.1519/JSC.0b013e318276b886 